\title{
TTR
}

Traduction, terminologie, re?daction

\section{La question dans les « textes bilingues » : analyse contrastive}

\section{Suzanne Pons-Ridler et Geneviève Quillard}

Volume 8, numéro 2, 2e semestre 1995

Technolectes et dictionnaires

URI : https://id.erudit.org/iderudit/037223ar

DOI : https://doi.org/10.7202/037223ar

Aller au sommaire du numéro

Éditeur(s)

Association canadienne de traductologie

ISSN

0835-8443 (imprimé)

1708-2188 (numérique)

Découvrir la revue

Citer cet article

Pons-Ridler, S. \& Quillard, G. (1995). La question dans les « textes bilingues » : analyse contrastive. TTR, 8(2), 197-210. https://doi.org/10.7202/037223ar

\section{Résumé de l'article}

La question dans les «textes bilingues " : analyse contrastive - Cette analyse a pour but de montrer dans quels domaines particuliers l'usage de la question diffère en anglais et en français. Elle se propose d'attirer l'attention des traducteurs sur les parties du discours que les francophones abordent spontanément par une forme interrogative et les anglophones par une déclarative, afin que les textes bilingues puissent comporter une proportion de questions plus conforme à celle que l'on a relevée dans les textes bilingues.
Tous droits réservés (C) TTR: traduction, terminologie, rédaction — Les auteurs, 1995 cest protége par la loi sur le droit d'auteur. L'utilisation des services d'Érudit (y compris la reproduction) est assujettie à sa politique d'utilisation que vous pouvez consulter en ligne.

https://apropos.erudit.org/fr/usagers/politique-dutilisation/ 


\section{La question dans les «textes bilingues»: analyse contrastive}

\section{Suzanne Pons-Ridler, Geneviève Quillard}

Cet article fait suite à une étude portant sur l'interrogation dans des revues de langue française et de langue anglaise ${ }^{l}$ (Pons-Ridler, Quillard, 1993). Nous avions constaté, en nous basant sur un corpus d'un millier de pages, que les journalistes francophones recouraient beaucoup plus fréquemment à la question et faisaient de l'interronégative un outil privilégié de persuasion.

Nous avons ensuite analysé des documents traduits du français en anglais et vice-versa. Cette étude préliminaire indiquait que le nombre d'interrogations était loin de passer du simple au double, mais elle montrait toutefois qu'il était toujours supérieur dans les textes rédigés ou traduits en français. Estimant qu'il s'agissait là de différences discursives qui méritaient qu'on s'y arrête, nous avons élargi notre corpus. Les données recueillies ont été classées selon qu'elles relevaient du domaine linguistique, de celui des rapports interpersonnels, de l'argumentation ou du style.

1. Numéros de mai et de novembre 1992 de L'Actualité, L'Express, Le Point et de The Economist, MacLean's, Time. Nombre total de pages: 570 en français, 562 en anglais. Nombre de questions affirmatives: 495 en français, 238 en anglais. Nombre d'interro-négations: 72 en français, 26 en anglais. Total des interrogations: 572 en français, 264 en anglais, soit $117 \%$ de questions de plus dans les revues de langue française. Pour un tableau plus détaillé, voir Interface, VIII(1), 1993, p. 44. 
Cette analyse a pour but de montrer dans quels domaines particuliers l'usage de la question diffère dans les deux langues. Elle se propose d'attirer l'attention des traducteurs sur les parties du discours que les francophones abordent spontanément par une forme interrogative et les anglophones par une forme déclarative, afin que les textes bilingues puissent comporter une proportion de questions plus conforme à celle que l'on a relevée dans les textes unilingues.

\section{Présentation du corpus}

Notre corpus comprend:

1. Des documents sur support informatique. Les questions ont été relevées et comptées par un programme informatisé.

a) Textes pragmatiques divers (articles de presse, bulletins d'information, circulaires, brochures): Français-anglais: 44031 mots, 57 interrogations en français, 45 en anglais. Anglais-français: 53172 mots, 70 interrogations en anglais, 81 en français.

b) Textes publicitaires, tous traduits de l'anglais au français: 9909 mots, 26 interrogations en anglais, 32 en français.

2. Des documents qui ne sont pas sur support informatique et qui totalisent un millier de pages: articles de revues bilingues comme En Route, Canadian, Forces, Atlas, Sentinelle, Langue et Société, Le Monde/Manchester Guardian, livrets publiés par le Secrétariat d'État, circulaires et brochures diverses. Étant donné que l'on ne peut guère se fier au comptage effectué à la main, cette partie du corpus n'a pas fait l'objet de statistiques. Mais nous en avons tiré un certain nombre d'exemples. À noter que la présentation de tous les exemples suit l'ordre texte-source/texte-cible.

\section{Commentaires}

Si la version française des documents de notre corpus comporte plus d'interrogations que la version anglaise, qu'il s'agisse du texte-source $(26,6 \%)$ ou du texte-cible $(15,7 \%)$, cette différence reste bien modeste quand on la compare à celle que nous avions relevée dans les revues unilingues $(117 \%)$. Bien que nos deux corpus (unilingue et bilingue) ne soient pas totalement comparables, puisque le second est moins homogène et que, pour reprendre une expression d'Antoine Berman, la 
part du «traduit» y est importante, cette différence est tout de même statistiquement importante et vient renforcer les résultats de notre première étude.

\section{Plan linguistique}

\section{Discours direct/indirect}

Le français marque un net penchant pour la forme directe ou indirecte libre, l'anglais pour la forme indirecte. Cette préférence du français pour l'interrogation directe s'explique dans un certain nombre de cas par des contraintes syntaxiques:

(1) Testimony in U.S. Superior court this week centered on whether the stipend Ms. Plaster Caster received 20 years ago was an advance against a planned book or a payment for her artistic rendering. / Les témoignages entendus au procès tournaient autour de la question suivante: les sommes reçues en 1969 par Mme Albritton étaient-elles une avance sur le livre projeté, ou un salaire pour son travail d'artiste? (The Globe and Mail, 23/4/03, L'Actualité, 7/93, p. 16)

(2) Les traductrices et traducteurs peuvent-ils jouer un rôle particulier ? / In answer to the question whether translators could play a special part (Circulaire, Réseau des traducteurs en éducation, 3/93)

(3) the real issue is whether individuals and institutions can adapt in time / la question fondamentale est la suivante: Pourrons-nous, en tant que particuliers et en tant qu'institutions, nous adapter assez rapidement à ces changements? (ACCC, International, automne 1991, p. 1)

On peut alors émettre l'hypothèse que le "pli» a en quelque sorte été pris et que, même lorsque rien ne s'oppose à l'utilisation du discours indirect, il vient moins spontanément au francophone que le discours direct ou indirect libre.

(4) «Comment voyez-vous l'avenir?», lui demanda-t-on. / When asked how he envisaged the future (Le Monde, 23/8/88; Manchester Guardian, 4/9/88)

Mais nous débordons alors le cadre strictement linguistique pour entrer dans le domaine de la rhétorique et de la stylistique.

Lorsque les propos sont rapportés au discours indirect, le français a tendance à les introduire sous une forme interro-négative, 
l'anglais sous une forme déclarative. Cette préférence du français pour les interro-négations ne saurait surprendre, étant donné la fréquence de ces formes dans les questions de la vie courante (Pons-Ridler, Quillard, 1991). Mais il faut également remarquer que le recours à l'interronégation vise à mieux assurer l'efficacité pragmatique de l'énoncé et relève donc aussi de stratégies argumentatives.

(5) Winston Churchill ne disait-il pas de la démocratie que c'était peut-être le pire des systèmes, à l'exception de tous les autres ? / As Winston Churchill said of democracy, it may be the worst of systems, with the exception of all the alternatives. (Langue et Société, n' 24, automne 1988, p. 14)

(6) Le roi ne dit-il pas en boutade qu'il est plus soucieux du bonheur national brut de ses sujets que du produit national brut ? I As the king likes to jest, he is more concerned with Gross National Happiness than with Gross National Product. (Atlas, 8/92, p. 128)

\section{Rapports interpersonnels}

\section{Impératif, interrogation et normes d'interaction}

Une analyse même rapide et superficielle de documents traduits montre que l'impératif (sous sa forme grammaticale, du moins) est beaucoup plus utilisé en anglais. «Il a été souvent noté [que] les locuteurs recourent en fait assez rarement à l'impératif [...], lui préférant des moyens plus détournés» (Kerbrat-Orecchioni, p. 200²). Lorsque l'impératif pourrait être perçu comme un ordre qui serait déplacé, sinon choquant (ce qui serait le cas si les exemples 7 et 8 avaient été traduits littéralement), étant donné qu'émetteur et destinataires sont sur un pied d'égalité et que leurs rapports sont loin d'être familiers's, les francophones remplacent cet acte de langage par un autre. La question,

2. Il est ici question de locuteurs francophones.

3. Jean Delisle note à ce sujet: "si elle est fréquente en français publicitaire, la forme impérative peut parfois produire des messages d'une brutalité choquante en français, comme c'est le cas de l'exemple suivant: Put cocktails back into your cocktail parties/ Vous promettez un cocktail. Alors servez-en.» (1993, p. 360) 
perçue comme moins menaçante pour la «face» du destinataire, figure en bonne place parmi les procédés de substitution.

(7) join this committee / pourquoi ne pas vous joindre à notre comité (circulaire, ATIO, 12/4/199)

(8) Get involved in starting one (a Papersave Program) / n'avez-vous jamais pensé à la possibilité de promouvoir vous-même la création d'un tel programme? (Forces canadiennes, Personnel Newsletter, 12/90, p. 16)

(9) Sort through the rat's maze of taxes, investment options and professional fees. / Le labyrinthe des impôts et des taxes, des possibilités de placement et des honoraires professionnels vous rebute ? (program, ATIO 1994 convention)

(10) Well, turn your (undivided) attention to these / Alors que diriez-vous de l'un de ces thèmes? (Canadian, 5/92, p. 12)

Cette réticence à utiliser le mode impératif se manifeste même lorsque celui-ci a une valeur incitative (ex. 11, 12), impersonnelle (13) ou lorsqu'il sert à présenter une requête (14).

(11) Imagine hot apple pie / Que diriez-vous d'une tarte aux pommes bien chaude (Actualités RAO, printemps 1991)

(12) A more constructive response is, "I know you are crying because you're upset. Let's talk about it." / Il serait plus constructif de répondre : «Je sais que tu pleures parce que tu as de la peine. Veux-tu qu'on en parle ?» (Brochure, Santé et Bien-être Canada, Comment aider les enfants à affronter la séparation)

(13) And let us not forget that all the rivers which have their source there have earned Guinea the reputation as the water tower of West Africa / La Guinée n'est-elle pas aussi qualifiée de «château d'eau de l'Afrique de l'Ouest» en raison des fleuves qui y prennent leur source ? (dans Jean Delisle, La traduction raisonnée, p. 422)

(14) Let me give you the profile, first, of our university's 17,000 full-time students. / Qui sont les 17000 étudiants qui fréquentent à temps plein l'université McGill ? (Langue et Société, n' 10, été 1983, pp. 32, 35)

Il faut d'ailleurs rappeler que, dans la langue parlée, l'interrogation sert souvent à exprimer un désir, une requête ou à donner un conseil. 
(15) On y va, Ordralphabetix ? / Come on, Unhygienix, let's go! (Astérix en Hispanie, Dargaud, 1969, p.25)

(16) Si on allait au cinéma ? / Let's go to the movies.

\section{Interrogation et inscription du destinataire dans le texte}

En présentant la communication comme une communication "in praesentia", l'interrogation établit entre le scripteur et le destinataire un système de relations discursives. L'attente suscitée par la question donne l'illusion d'un contact, d'un dialogue: «le style devient moins impersonnel» (Colson, p. 75).

Étant donné la fréquence nettement supérieure des formes interrogatives en français, il y a lieu de penser que la «tendance du français à ne pas marquer la relation inter-énonciateur) (GuilleminFlesher, p. 65) ou "dépersonnalisation du message» (Delisle, 1993, p. 360 ) est beaucoup moins prononcée qu'on ne l'a cru jusqu'à ce jour. Certes, les formes impersonnelles ont longtemps été la norme dans certains types de discours (recettes, modes d'emploi, notices d'entretien, guides de l'utilisateur, instructions à suivre, etc.), encore que l'usage moderne ait de plus en plus tendance à les personnaliser. Il est également vrai que l'utilisation du pronom personnel you donne parfois aux écrits «un ton qui peut paraître familier à un lecteur francophone» (Delisle, 1993, p. 360) et que ce dernier évite alors une formulation aussi directe. Mais il existe bien d'autres manières d'inscrire le destinataire dans un texte, et la question est l'une d'entre elles, puisqu'elle donne au destinataire l'impression de participer activement à l'acte communicatif.

(17) Le thème de cette pièce de quatre-vingt-dix minutes ? L'être humain et ses sentiments / The ninety-minute piece is about human beings and their feelings (Atlas, 3/94, p. 53)

(18) Notre volonté ? Vous offrir les meilleurs produits de soins / Our aim is to offer you the highest quality beauty products (Carte cliente Clarins)

La question permet aussi d'adoucir l'impression de familiarité que l'inclusion du pronom personnel apporte en français et de faire accepter plus facilement son emploi. Nombreux sont les exemples qui 
combinent question et pronoms personnels dans des formulations qui ne risquent pas de heurter le destinataire.

(19) This may come as a surprise to sailing enthusiasts / ca vous étonne ? (En Route, 6/90, pp. 30, 34)

(20) Savez-vous pourquoi, depuis plus de vingt ans, Frédéric François traverse les modes musicales sans lâcher la rampe du succès ? / For more than twenty years Frédéric François has been successful in every musical genre. (Atlas, $3 / 94$, p. 52)

(21) Here's what happens with a pair of US-made earrings that costs the equivalent of $C \$ 500$. / Voulez-vous connaitre le destin d'une paire de boucles d'oreilles fabriquées aux États-unis qui coûtent l'équivalent de 500 \$ CAN? (En Route, 7/90, p. 14)

\section{Argumentation}

\section{1. Évidences et questions rhétoriques}

Une question rhétorique est une question dont la réponse est évidente et qui «prétend forcer le destinataire à reconnaître explicitement ou non, ce que le locuteur tient pour vrai» (Ducrot, 1977, p. 28) ${ }^{4}$. Elle a donc essentiellement une fonction argumentative et elle constitue une forme privilégiée en français pour présenter une évidence, ou ce que le scripteur veut faire passer pour telle. En revanche, comme l'ont noté grammairiens et traducteurs (Leech et Svartik, 1980, p. 27; Vinay et Darbelnet, 1958, p. 218; Darbelnet, 1978, p. 136; Newmark, 1988, p. 64), l'anglais ne la prise guère, préférant les formes déclaratives, qui sont beaucoup moins percutantes sur le plan argumentatif. Étant donné que ne pèse ici aucune contrainte linguistique, il s'agirait là d'une différence essentiellement culturelle. Le texte français généralise davantage, alors que le texte anglais reproduit plus fidèlement la réalité et fait plus souvent appel à des modalisateurs.

4. On trouve déjà pratiquement la même définition chez Fontanier (p. 368): «L'Interrogation consiste à prendre le tour interrogatif, non pour marquer un doute et provoquer une réponse, mais pour indiquer, au contraire, la plus grande persuasion, et défier ceux à qui l'on parle de pouvoir nier ou même répondre.) (Les italiques sont de l'auteur.) 
(22) Cliché immuable, inséparable de la Bretagne, côté vacances : combien d'enfants n'ont-ils pas pêché, haveneau à la main, dans les trous d'eau ? / A fixture of holidays in Brittany, and a treasured childhood memory for countless adults : shrimping in the rock pools. (Atlas, 3/94, p. 127)

(23) N'a-t-on pas retrouvé des traces de leur (les Vikings) passage sur les côtes d'Amérique cing siècles avant la traversée de Christophe Colomb ? / Archeological evidence shows that they even fetched up on the coasts of America, five centuries before Columbus. (Atlas, 8/02, pp.88, 89)

(24) N'est-il pas logique qu'à cet esprit sportif, les Québécois joignent leur hospitalité incomparable? / As might be expected of people who enjoy all this, les Québécois are hospitable hosts. (Plaisir d'hiver au Québec, Ministère du tourisme, de la chasse et de la pêche, p. 2)

La question rhétorique est souvent un stratagème permettant de présenter sous forme de vérité universelle et irréfutable ce qui n'est en fait que l'opinion ou la réaction de l'émetteur. Ainsi dans l'exemple 25 la traduction change radicalement la portée du message: «la question porte moins sur la recherche d'un motif que sur la recherche de la raison pour laquelle on n'en trouvera pas; elle est surtout affirmation qu'il n'y a pas de motif suffisamment explicatif» (Perelman, OlbrechtsTyteca, pp. 214-215).

(25) How you can live next door to a family for 10 to 15 years, then try and kill them is beyond me. / Comment pouvez-vous avoir un voisin pendant 10 ou 15 ans pour ensuite tenter de l'assassiner? (Sentinelle, 10-11/92 p. 6)

(26) Un étudiant qui affirme "hair le FN» ne l'a-t-il pas choisie comme directeur de mémoire? / A student who claims to hate the FN chose her as his thesis superviser (Le Monde, 18/5/90; Manchester Guardian, 26/6/90)

Le manque d'attirance des anglophones pour les questions oratoires semble être confirmé par le fait qu'elles sont parfois tout simplement éliminées dans les traductions. Certes, il peut peser sur le traducteur certaines contraintes d'ordre matériel (mise en page notamment), mais n'est-il pas révélateur que le couperet s'abatte surtout sur les questions rhétoriques?

(27) Comment faire découvrir la musique des Latins aux Hongrois ? (ADP magazine, 6/94)

(28) Comment alors pourrait-on faire fi de la culture ? (Bulletin, Commission canadienne pour l'Unesco, 6/87, p.1) 


\section{Abolition des distances entre émetteur et destinataire}

En abolissant les distances entre le je et le tu, la question vise à contraindre le destinataire à partager l'idée ou le jugement de l'émetteur et joue un rôle majeur dans l'argumentation. Le destinataire est invité à réfléchir et il est souvent guidé par d'autres questions qui jalonnent le raisonnement, ce qui a pour but de le faire adhérer plus facilement aux arguments avancés. En fait, l'interrogation est alors un "procédé assez hypocrite pour exprimer certaines croyances» (Perelman, OlbrechtsTyteca, p. 214) et les faire partager au lecteur. Les exemples 29 et 30 sont à cet égard très révélateurs. La modalisation des énoncés anglais leur donne un tout autre ton que leur «équivalent» français.

(29) We think you'll agree that it will give you a considerable advantage over your competition. / Bel avantage sur vos concurrents, n'est-ce-pas? (publicité, Grid, 1992)

(30) If there is room in your life for that kind of reliability, put us to the test. / Vous recherchez justement une telle fiabilité ? Choisissez UPS. (publicité, UPS, Time, 22/12/93, l'Actualité, 1/12/93)

(31) en serait-il ainsi de l'isotherme de $10 \mathrm{C}$ en juillet ? / this might be the case for the 10C isotherm in July (L.-E Hamelin, Le Nord canadien et ses référents conceptuels, Secrétariat d'État du Canada, 1988, p. 18)

\section{Effacement apparent du scripteur}

La question permet aussi au scripteur de s'effacer, en apparence du moins, de ne pas paraître imposer son point de vue au lecteur. Mais tout en incitant le destinataire à réfléchir et à fournir lui-même une réponse, la question est formulée de telle sorte qu'elle limite le cadre des réponses possibles. Il s'agit là d'une stratégie argumentative, car «nême s'il ne conclut pas, le locuteur de question la présente comme orientant le discours vers certains types de conclusions, et à l'exclusion des autres» (Anscombre, Ducrot, p. 137).

(32) proving, perhaps, that those who can, can teach as well as do / Ne seraitce pas la preuve que ceux qui savent, savent enseigner aussi ? (En Route, $12 / 94$, pp. 14,16 )

(33) The curse of Cartier's time has been lifted. / La malédiction datant de l'époque de Cartier viendrait-elle d'être conjurée ? (Canadian, 2/1993, p. 41) 
(34) Et si Reims osait ne plus vivre seulement à l'abri de sa merveilleuse cathédrale gothique ? / As if Rheims dares to exist in another way, not only sheltered by its marvellous Gothic cathedral. (Demeures et châteaux, No 54, mars-avril 1990, pp. 25, 26)

\section{Ironie, outil d'argumentation dans l'interrogation}

Pour rendre les arguments plus percutants, le scripteur peut les charger d'ironie. $\mathrm{Si}$, de plus, il utilise une forme interrogative, il établit une certaine connivence avec le destinataire, l'ironie étant en quelque sorte partagée par les partenaires de l'acte de communication, ce qui n'est pas le cas lorsque l'observation est présentée sous une forme déclarative.

(35) En même temps, les professionnels du cinéma redécouvrent - un peu tard? / At the same time, film directors and scriptwriters, wracked by self-doubt (rather late in the day, it has to be said) (Le Monde, 23/8/1988, Manchester Guardian, 4/9/1988)

(36) comment ne pas remarquer que Georges Pinault a été nommé maître de conférences associé de celte ancien ? / it is no coincidence that Georges Pinault was appointed associate lecturer in Celtic (Le Monde, 18/5/90; Manchester Guardian, 24/6/90)

\section{Plan stylistique}

Sur le plan stylistique, elle joue un rôle capital, car elle permet de varier le rythme et les structures phrastiques et d'éviter ainsi une certaine monotonie. Or les francophones sont littéralement hantés par tout ce qui risquerait de paraître trop plat ou répétitif. D'où les "pourquoi», les «qui sait», les "comment», les interrogations de toutes sortes qui émaillent leurs écrits. Il faut également remarquer que la question permet aussi fréquemment d'éviter l'utilisation des verbes avoir ou être, ce qui constitue évidemment un précieux atout.

Une question fondamentale se pose ici: quel est le rapport entre le code linguistique et la stylistique? Est-ce parce que le français est une langue beaucoup plus rigide et contraignante sur le plan morphosyntaxique qu'il importe plus de varier les tournures, d'éviter les répétitions, de changer de rythme, etc.? 
(37) Laisser à l'abandon une année complète de travail préparatoire ? Il n'en était pas question. / the stakeholders were loath to let a full year of preparatory week go to waste (InformAT7O, vol. 22, ñ 3,6/93, pp. 2,4)

(38) Now, instead of harmony, mostly divisiveness flows from Meech Lake / Oủ en est l'harmonie? L'accord du lac Meech n'est plus que source de division (Langue et Société, $\mathrm{n}^{\circ} 24$, Automne 1988, p. 40)

(39) Le thème de cette pièce de quatre-vingt-dix minutes ? L'être humain et ses sentiments / The ninety-minute piece is about human beings and their feelings (Atlas, 3/94, p. 53)

(40) If you've never heard of fricot, poutines rapées or chiard, you're not alone. / Vous n'avez jamais entendu parler de fricot, de poutines râpées ni de chiard ? Vous n'êtes pas les seuls. (En Route, 10/88, pp. 80, 81)

(41) Le crime de tous ces responsables? Avoir entrouvert la Bulgarie sur une vision moins figée des choses et de l'Histoire / Their crime was to have given Bulgaria a glimpse of a vision of things and history less frozen in time (Le Monde, 24/25-7-88; Manchester Guardian, 31-7-88)

(42) What we finally realized was that, wherever you want to sell, you tailor your consumer communications to the local culture. And you can best do that by having your advertising designed by people who live in that culture. / Finalement il nous vint a l'esprit que quiconque veut vendre un produit doit teinter sa publicité de couleur locale. Comment? Tout simplement en retenant les services d'un publicitaire dont l'identité culturelle est celle du consommateur-cible. (Langue et Société, n"10, Été 1983, pp. 23, 25)

(43) The result is a completely redesigned car. / Résultat ? Une voiture entièrement redessinée. (publicité, Jetta, Time, 18/10/1993, L'Actualité, 15/10/93)

\section{Conclusion}

Les catégories que nous avons établies dans cette étude ne sont pas étanches, loin de là, l'interrogation jouant souvent un rôle sur plusieurs plans à la fois, mais elles permettent de montrer, d'abord qu'il existe un rapport entre la forme interrogative en français et certaines contraintes linguistiques, ensuite que les interrogations remplissent des fonctions diverses. En ce qui concerne les relations inter-énonciateurs, elles inscrivent le destinataire dans l'énoncé, lui donnant ainsi l'impression qu'il est un partenaire actif de l'acte de communication; elles constituent donc un moyen de personnaliser le message, alors qu'en anglais ce sont surtout les pronoms personnels qui assument cette fonction. Sur le plan 
argumentatif, elles jouent un rôle capital, l'abolition des distances entre destinateur et destinataire étant pour celui-là un moyen d'imposer son point de vue ou de parvenir à ses fins. Sur le plan stylistique, enfin, elles permettent de varier les structures phrastiques et, dans le cas des interrogations averbales, de rendre le rythme plus rapide.

Il faut remarquer, à en juger par l'écart qui sépare les chiffres obtenus à partir de ce corpus et du corpus unilingue dont nous nous sommes servies pour notre précédente étude, et tout en tenant compte des différences typologiques que présentent les deux corpus, que les formes interrogatives sont probablement sous-représentées dans les traductions de l'anglais au français et sur-représentées dans les traductions du français à l'anglais. Or, si l'«on exige précisément du traducteur qu'il sache (re)composer un texte comme s'il s'agissait d'une rédaction originale» (Delisle, 1984, pp. 217-218), il faudrait que, par le biais d'observations et d'exercices appropriés, l'on incite les apprentistraducteurs à produire des textes-cibles qui reflètent davantage les pratiques discursives de la communauté à laquelle ils s'adressent. On éviterait ainsi des formulations pour le moins surprenantes, telles que:

Mais, pour savoir s'affranchir des contraintes, ne faut-il pas déjà... être libre? / But to abandon constraints, one should already be free, no? (Demeures et chateaux, Inventaire 5/90, pp. 53, 54)

Suzanne Pons-Ridler, Department of Humanities and Languages, University of New Brunswick, PO Box 5050, Saint John, New Brunswick, Canada E2K 3S7

Geneviève Quillard, Royal Military College, Kingston, Ontario, Canada K7K 5LO

\section{Références}

ANSCOMBRE, Jean-Claude et Oswald Ducrot (s. d.). L'argumentation dans la langue. Bruxelles, Pierre Mardaga.

COLSON, Jacques (1989). Le Dissertoire. Bruxelles, De Boeck. 
DARBELNET, Jean (1978). «Systèmes oppositionnels en français et en anglais», Babel, XXXIII(3-4), pp. 135-138.

DELISLE, Jean (1984). L'analyse du discours comme méthode de traduction. Ottawa, Éditions de l'Université d'Ottawa.

(1993). La traduction raisonnée. Ottawa, Les Presses de l'Université d'Ottawa.

DUCROT, Oswald (1977). «Illocutoire et performatif», Linguistique et Sémiotique. L'illocutoire. Travaux du Centre de rechercheslinguistiques et sémiologiques de Lyon, pp. 17-55.

FONTANIER, Pierre (1977[1821-1830]). Les figures du discours. Paris, Flammarion.

GUILLEMIN-FLESHER, Jacqueline (1986). «Le linguiste devant la traduction", Fabula. Traduire, Presses Universitaires de Lille, pp. 59-69.

KERBRAT-ORECCHIONI, Catherine(1992). Les interactionsverbales, tome II. Paris. Armand Colin.

LEECH, Geoffrey et Jan Svartik (1980). A Communicative Grammar of English. London, Longman.

NEWMARK, Peter (1988). A Textbook on Translation. Prentice-Hall International (UK).

PERELMAN, Ch. et L. Olbrechts-Tyteca (1983). Traité de l'argumentation. La nouvelle rhétorique. Éditions de l'Université de Bruxelles.

PONS-RIDLER, Suzanne et Geneviève Quillard (1991). "Stylistique comparée: la forme interrogative en français et en anglais», $L a$ linguistique, $\mathrm{n}^{\circ}$ 1. Paris, P.U.F., pp. 111-118.

(1993). «L'interrogation dans les textes écrits. Fréquence et fonctions en anglais et en français», Interface, VIII(1), pp. 43-57. 
VINAY, Jean-Paul et Jean Darbelnet (1958). Stylistique comparée du français et de l'anglais. Montréal, Beauchemin.

RÉSUMÉ: La question dans les «textes bilingues»: analyse contrastive - Cette analyse a pour but de montrer dans quels domaines particuliers l'usage de la question diffère en anglais et en français. Elle se propose d'attirer l'attention des traducteurs sur les parties du discours que les francophones abordent spontanément par une forme interrogative et les anglophones par une déclarative, afin que les textes bilingues puissent comporter une proportion de questions plus conforme à celle que l'on a relevée dans les textes bilingues.

ABSTRACT: Using the Question in English and French: a Contrastive Analysis - This article shows the areas in which English and French depart in their usage of interrogation. It brings to the translator's attention the discursive contexts where francophones tend to use an interrogative and anglophones a declarative sentence. This will help maintain in translations a proportion of these forms more consistent with the patterns observed in original French or English texts. 